\title{
Differential relational encoding of categorical information in memory for action events
}

\author{
JOHANNES ENGELKAMP, KERSTIN H. SEILER, and HUBERT D. ZIMMER \\ Saarland University, Saarbrücken, Germany
}

\begin{abstract}
Memory for action phrases is better if the actions are enacted in subject-performed tasks (SPTs) than if they are only listened to in verbal tasks (VTs). This effect is ascribed to better item-specific encoding of SPTs than of VTs. The role of interitem relational information is controversial, and the findings of clustering with categorically structured lists are inconsistent (see Engelkamp, 1998). The present study contributes to clarifying these effects by demonstrating that intentional relational encoding can be used more efficiently in VTs than in SPTs and influences the degree of clustering. If the list structure is not obvious, inducing intentional encoding by presenting the category labels prior to list presentation and asking subjects to use this preinformation increases clustering in VTs but not in SPTs. Without preinformation, clustering scores of VTs and SPTs did not differ; with preinformation, clustering of VTs was stronger than that of SPTs. The authors suggest how the inconsistent findings with regard to clustering effects can be explained.
\end{abstract}

Often, what one remembers refers to things that one has done. One taps this kind of memory, for instance, when one describes to another person what activities one has carried out in the course of a day. This interest in one's activities contrasts with traditional memory research, in which mainly memory for verbal material is studied. Therefore, an important line of new memory research emerged when, about 25 years ago, researchers began to study memory for action events (Cohen, 1981, 1983; Engelkamp \& Krumnacker, 1980; Saltz \& DonnenwerthNolan, 1981). Since that time, a fair amount of research has been devoted to memory for action events (see Engelkamp, 1998; Nilsson, 2000; or Zimmer, 2001, for reviews). In the typical research paradigm of action memory, subjects are required to enact a series of action phrases such as "peel the banana" or "break the stick." They may use real objects or, as is usually the case, imagine using real objects when the actions require objects. Memory for such subject-performed tasks (SPTs) is usually compared with memory for verbal tasks (VTs), in which subjects only listen to or read the phrases.

The central finding from such comparisons, called the $S P T$ effect, is that recall and recognition memory are better for SPTs than for VTs. Because a number of variables had different effects on performance in the two tasks, different explanations were proffered, including the proposals that memory for actions follows different laws than does verbal memory (Cohen, 1985), that memory for ac-

This research was supported by Grant En 124/13 from the Deutsche Forschungsgemeinschaft to J.E. and H.D.Z. We thank Nicola Ferdinand, Jochen Glössner, Hedda Janssen, and Annabell Saffran for their assistance with data collection. Correspondence concerning this article should be addressed to J. Engelkamp or H. D. Zimmer, FR Psychologie, Saarland University, D-66123 Saarbrücken, Germany (e-mail: engelkamp@mx. uni-saarland.de or huzimmer@mx.uni-saarland.de). tion events is multimodal and particularly rich (see, e.g., Bäckman, Nilsson, \& Chalom, 1986), and that a specialized action output system is involved in SPTs but not in VTs (Engelkamp \& Zimmer, 1983). In spite of all these different proposals to explain the findings related to action memory, it was widely accepted that the distinction between item-specific and relational information could make a valuable contribution to explaining said findings (Engelkamp, 1988; Knopf, 1991; Kormi-Nouri, 1995; Zimmer, 1991). However, the role that relational encoding processes plays in action memory remained controversial. The main goal of this study is to help clarify the role of relational encoding processes. In doing so, we will also contribute to the understanding of relational processes in verbal and in nonverbal tasks in general, as well as to exploration of the question of whether and how relational processes may influence the level of recall.

\section{Item-Specific and Relational Information}

The literature quite strongly supports a separation between memory of item-specific information and memory of relational information in list learning (see, e.g., Burns, 1993; Chalfonte \& Johnson, 1996; Gillund \& Shiffrin, 1984; Hunt \& Einstein, 1981; McDaniel, Einstein, Dunay, \& Cobb, 1986; Mulligan, 1999; Zimmer, 1991). Itemspecific information refers to the information that is specific for each individual item and that allows the item to be reintegrated and discriminated from other items. In contrast, relational information refers to associations among items. It serves particularly to support search processes in memory. Relational interitem associations can be formed along different dimensions. In the present study, the focus is on categorical organization.

Categorical knowledge is available in abstraction hierarchies of objects and events. Among other things, se- 
mantic memory is organized along such knowledge structures. Although relational encoding and retrieval need not be based on intracategorical associations, this type of relational information has been studied often since Bousfield (1953) discovered the categorical clustering effect. The clustering effect is the phenomenon in which items belonging to the same category tend to be recalled together even if they were not presented together during study. One reason for studying categorically structured lists is that with such lists the degree of categorical clustering in recall can be measured in terms of clustering scores (see, e.g., Gollin \& Sharps, 1988; Hunt \& Einstein, 1981; McDaniel, Einstein, \& Lollis, 1988; Mulligan, 2001; Ritchey, 1980; Zimmer, 1989). Categorical lists are used in the present study.

\section{Categorical Relational Information in Memory for Action Events}

With regard to action memory, most researchers agree that SPTs provide much better item-specific information than VTs do, and they attribute the SPT effect to this advantage of SPTs (see, e.g., Engelkamp \& Zimmer, 1997; Helstrup, 1987; Kormi-Nouri, 1995; Saltz \& DonnenwerthNolan, 1981). However, the question of whether enactment also improves the interitem relational information in comparison with VTs is controversial (see, e.g., Bäckman et al., 1986; Engelkamp \& Zimmer, 1996; Kormi-Nouri \& Nilsson, 1999). The controversy is based largely on findings from categorically structured lists and on the adjusted ratio of clustering (ARC) score (Roenker, Thompson, \& Brown, 1971) as an index of relational encoding. ${ }^{1}$ This score is assumed to measure the degree to which a subject's recall follows the categorical structure of the list as indicated by the degree to which the subject recalls same-category items together. The higher the ARC score, the better subjects are assumed to have processed the categorical information and the more they are assumed to have used it during recall.

Bäckman et al. (1986) assumed that the multimodal nature of the actions required during SPTs generally facilitates and enriches encoding. This should provide not only more item-specific information than is provided by VTs, but better relational information as well. Bäckman et al. did not distinguish between different types of relational information. Their position was supported by their finding that the ARC scores were higher for SPTs than for VTs. In contrast, Engelkamp and Zimmer (1994) focused on the motoric nature of SPTs and assumed that more item-specific information should be provided by SPTs than by VTs, but that the degree of categorical relational information should not differ between SPTs and VTs. The key argument for the claim of better itemspecific information in SPTs was that enactment forces subjects to focus more on the individual items than does verbal learning (see, e.g., Engelkamp, 1995; Zimmer \& Engelkamp, 1999) and that encoding of action-specific features enhances the representation of each item (Zimmer, 2001). Categorical relational information should not differ between SPTs and VTs because it is based on preexperimental conceptual knowledge and is encoded by the process of spreading activation among the categorically related representations. In fact, in several studies no differences were observed in the ARC scores for VTs and SPTs (Engelkamp \& Zimmer, 1996; Zimmer \& Engelkamp, 1989). Engelkamp and Zimmer (1994) assumed that the same processes that underlie semantic priming effects also underlie the categorical clustering effects in free recall. Recently, Roediger, Balota, and Watson (2001) also suggested that spreading activation may be extended to episodic memory paradigms. Therefore, it seems reasonable to assume that activation spreading can underlie categorical clustering effects. Moreover, according to Engelkamp and Zimmer (1994), categorical relational encoding processes in the conceptual system should be independent of the motoric nature of SPTs.

If we assume that SPT instructions focus on single actions and hinder the use of intentional relational encoding, then, with related lists, any categorical relational encoding should be based only on spreading activation among the related concepts. There should be little difference between results following explicit instructions to use the categorical information intentionally and results when no such instructions are provided. In contrast, such instructions may be helpful with VTs because they allow subjects to direct their attention to the categorical information.

Whether instructions to use categorical information will be helpful in VTs depends on whether or not the categorical information is already used without such instructions. Without explicit instructions, the categorical structure is more likely to be used as the list structure becomes more obvious. For instance, if categorical lists are presented blocked along the categories, then spreading activation already leads to an efficient clustering and intentional processes can add little more. Engelkamp and Zimmer (2002, Experiment 2) and Engelkamp, Seiler, and Zimmer (in press, Experiment 2) have shown that under such conditions ARC scores for both VTs and SPTs are high and do not differ. However, the situation should be different if the list structure is not immediately obvious, as is often the case when the items from the categories are randomly presented. With random presentation in VTs, either of two things may happen: First, subjects' intentional associative processes may be congruent with the categorical list structure. In this case, their ARC scores might be higher than those in SPTs. Second, their intentional associations may deviate from the experimenterdefined list structure. In this case, the ARC scores in VTs may be lower than those in SPTs. Empirically, the most frequent finding is that ARC scores of VTs and SPTs do not differ. This is the case in 9 of 11 experiments reported in the literature (see Engelkamp et al., in press; Engelkamp \& Zimmer, 1996, 2002; Norris \& West, 1993; Zimmer \& Engelkamp, 1989). In the two remaining experiments, the ARC scores were higher for SPTs than for VTs (Bäckman et al., 1986, Experiment 1; Engelkamp et al., in press, Experiment 2). These find- 
ings could be explained if it is assumed that in VTs some subjects usually generate more interitem associations that are congruent with the list structure and some subjects generate more incongruent associations. Therefore, the group average scores of VTs and SPTs usually do not differ. This speculation would also explain why sometimes even substantial mean ARC score differences were not significant.

If these considerations hold, presenting subjects the categorical labels and asking them to use them to encode the items relationally should help the subjects to do so in VTs but not in SPTs. Assuming that SPTs allow little intentional encoding, their clustering should be largely independent of explicit instructions to encode relational information intentionally. Assuming that VTs allow flexible and intentional encoding, clustering in VTs should increase with explicit instructions to encode the items along the categories, provided the categories are not immediately obvious. This increase should have the additional consequence that with such an instruction clustering in VTs should be higher than clustering in SPTs.

Hints that this might happen come from a former and hitherto neglected observation of Zimmer and Engelkamp (1989). They studied episodically and taxonomically structured lists of action verbs in VTs and SPTs. The episodic list consisted of 24 action verbs from six everyday episodes, such as "to do carpentry." The taxonomic list consisted of 40 action verbs from eight taxonomies, such as "to clean something." In their Experiment 1, they presented the list items in a random order under an unspecific memory instruction. In their Experiment 2, they presented the same lists again in random order, but now the subjects were given the category labels in advance and were explicitly instructed to categorize the items during encoding in order to enhance memory performance. In both experiments, the lists were presented twice with a free recall after each presentation. On the first trial, the results showed that the ARC scores were higher when preinformation about the categories of the list was presented only after VTs. In addition, higher ARC scores were observed in VTs than in SPTs when the subjects were preinformed about the categories. Because this interaction was not significant and the effect of the categorization instructions on VTs and SPTs occurred across experiments, we did not consider this difference to be important at that time. However, these results are in line with the theoretical ideas developed above and, therefore, we decided to examine them here.

\section{Item and Relational Information and Free Recall Performance}

According to some (e.g., Einstein \& Hunt, 1980; Hunt \& Einstein, 1981; McDaniel et al., 1986; Zimmer, 1991), free recall is based on a generation process, the efficiency of which is determined by relational information, and on a subsequent recognition process, the efficiency of which is determined by item-specific information. The latter process allows one to decide whether a gener- ated item is old or new. Important to this position is the assumption that the recall depends on both types of information. How a manipulation influences recall depends on how both types of information are related. If there is a tradeoff between relational and item-specific information, an increase of one type would lead to a decrease of the other type. Under this assumption, free recall should be optimal if both types of information are in balance. The more the emphasis on one type of information over the other, the poorer recall performance will be. A review of the literature indicates that evidence of an encoding tradeoff between item-specific and relational information is equivocal (see, e.g., Hockley \& Cristi, 1996). In many of the studies designed to test the tradeoff hypothesis, a paired-associate learning paradigm was used and memory of relational information was tested either by cued recall or by pair recognition memory. In contrast, item-specific information was tested in recognition memory for single items. The findings of list learning are also equivocal with regard to the tradeoff assumption. Biegelmann (1994) and Engelkamp, Biegelmann, and McDaniel (1998), for instance, could not support the tradeoff assumption, whereas Hunt and Seta (1984) reported findings in line with the assumption.

Because the data do not consistently support either the tradeoff assumption or the assumption of independence of both types of information, it is difficult to predict the level of recall performance from variable organizational scores. Assuming that categorical relational information could be varied independently of item-specific information, the degree of relational information should correlate positively with performance in free recall. However, such positive correlations between free recall and ARC scores in studies of action memory are not always observed (see, e.g., Engelkamp \& Zimmer, 1996; Zimmer \& Engelkamp, 1989). If anything, the relationship between free recall and ARC scores seems to hold for VTs rather than for SPTs. Moreover, the tradeoff assumption suggests that raising the ARC level of VTs should increase free recall of VTs, and the SPT effect in free recall should be reduced or disappear. However, recent findings have shown that the SPT effect was independent of an increasing ARC score (see, e.g., Engelkamp et al., in press).

Such results are less problematic if one considers more recent theorizing, which suggests that different retrieval strategies may underlie free recall. Depending on the degree to which relational information and item-specific information are available, it is claimed that a search-based or distinctiveness-based retrieval strategy dominates (Engelkamp \& Dehn, 2000; Greene, 1992; McDaniel, DeLosh, \& Merritt, 2000; Zimmer, Helstrup, \& Engelkamp, 2000). A distinctiveness-based retrieval strategy should dominate if item-specific information is easily available, and a search-based strategy should dominate if there is strong relational information. Zimmer et al. showed that recall of SPTs is dominated by distinctiveness-based retrieval, which is reflected in an enhanced recency ef- 
fect and a weakened primacy effect. In contrast, VTs appeared to be dominated by search-based retrieval, which is reflected in a pronounced primacy effect and a short recency effect. ${ }^{2}$ Seiler and Engelkamp (2003) were able to support this view further. They showed that an item-specific orienting task with VTs enhanced the recency effect and made the primacy effect disappear. Moreover, the comparison of VTs under standard and item-specific instructions with unrelated lists showed a reduced freerecall performance under item-specific instructions. Because in other studies item-specific instructions were always compared with relational instructions (e.g., Hunt \& Einstein, 1981; McDaniel et al., 1988) and did not include a standard learning instruction, they could not observe this effect. Seiler and Engelkamp speculated that this finding indicates that recall of VTs depends on both item-specific and relational information.

These considerations and findings make it difficult to predict the level of free recall with varying degrees of relational information. Because the retrieval processes seem to be adaptive to the item-specific and relational information available, the level of free recall should tend to be relatively stable. Hence, we do not expect substantial variations in the level of free recall with varying ARC scores. For the same reason, we predict that the $\mathrm{SPT}$ effect will remain stable even if the ARC score varies.

To test our predictions about the ARC scores in VTs and SPTs, we conducted two experiments. Experiment 1 was designed to assess whether higher ARC scores for VTs than for SPTs, such as those reported in Experiment 2 of Zimmer and Engelkamp (1989), was a replicable effect. Because the pattern was indeed replicated, we conducted Experiment 2 with a new set of items to assess the effects of preinformation about list categorization on both ARC scores and free recall.

\section{EXPERIMENT 1}

The main goal of Experiment 1 was to determine whether ARC scores in VTs are indeed greater than those in SPTs if preinformation about the list structure is given in advance. This experiment was a partial replication of Experiment 2 of Zimmer and Engelkamp (1989).

\footnotetext{
Method

Subjects. The subjects were 48 students of Saarland University. Half of them learned the list in VTs, and half learned it in SPTs.

Materials. The study list consisted of 40 action verbs from eight categories, such as "cleaning" and "eating," which were taken from Zimmer and Engelkamp (1989). Five items were presented from each category. For example, the items POLISH, BRUSH, and WASH belonged to the category cleaning. The items were presented in random order with the restriction that any two items of the same category were separated by at least two other items.

Procedure. Half of the subjects were instructed to learn the list of verbs by just listening to them (VT), and the other half were instructed also to mimic the denoted actions (SPT). In addition, before the list was read to the subjects, all were given the category labels and explicitly requested to use the categories during encoding to assign the items to them, because to do so would enhance their
}

memory performance. They were also informed before list presentation that a recall test, in which they would have to write down as many verbs as possible, would follow immediately after list presentation. The items were read aloud to the subjects at $5-\mathrm{sec}$ intervals.

\section{Results and Discussion}

As was expected, there was an SPT effect. The subjects recalled more items after SPTs (.46) than after VTs $[.33 ; t(46)=-3.86, S E=.0265, p<.001]$. More important, the ARC scores showed the inverse result: They were higher after VTs $(.56)$ than after SPTs $[.31 ; t(46)=$ 2.69, $S E=.0758, p<.01]$.

These findings show that more efficient use of intentional relational processes is made in VTs than in SPTs if subjects are preinformed about the list and requested to use a strategy that guarantees that the encoding processes correspond to the categories of the list. Hence, this experiment replicates the observations of Zimmer and Engelkamp (1989, Experiment 2). Moreover, it demonstrates that intentional relational encoding processes can change the predominant findings that ARC scores do not differ for VTs and SPTs in categorically structured lists (see, e.g., Engelkamp \& Zimmer, 1996; Norris \& West, 1993; Zimmer \& Engelkamp, 1989) or are lower in VTs than in SPTs (see, e.g., Bäckman et al., 1986; Engelkamp et al., in press).

The finding that despite higher ARC scores in VTs than in SPTs there was still the usual recall advantage of SPTs demonstrates moreover that relational information is not the basis of the SPT effect and that there must be other retrieval strategies than generation and recognition. This is in line with the assumption that item-specific information is decisive for the SPT effect.

\section{EXPERIMENT 2}

The main purpose of Experiment 2 was to assess whether or not there is an interaction in ARC scores between type of task (VT vs. SPT) and preinformation about the categorical list structure (with vs. without preinformation) but no interaction in free recall. In order to generalize the findings and to relate them to the studies by Bäckman et al. (1986), Engelkamp and Zimmer (1996), and Norris and West (1993), we used action phrases as items and a list structure based on taxonomies of the objects used in the action phrases.

\section{Method}

Subjects. One hundred eight persons took part in this experiment. They were all students of Saarland University and were paid for their participation. Half of them were randomly assigned to the VT condition and half to the SPT condition. Half of the subjects in each condition were preinformed about the categories of the list, and half were not. Hence, there were four groups of 27 subjects.

Materials. As learning material, three different lists were used, each consisting of 42 action phrases comprising an action verb and an object. In an effort to get data that were less dependent on the accidental items than would be the case if only one list were used, three different lists were used. One of the three lists was presented to each subject, and the lists were balanced over conditions. There were six categories, each containing seven phrases. The categories 
were created along the taxonomic structures of the objects used in the actions. For instance, the category food consisted of phrases such as "to grate the cheese," "to salt the potatoes," and "to crack the egg." This list structure was used because it had been used in most of the other studies from the literature (e.g., Bäckman et al., 1986; Engelkamp \& Zimmer, 1996; Norris \& West, 1993). During presentation, the items of the different categories appeared in a pseudorandom order. The sequence was randomly generated with the constraint that any two items of the same category had to be separated by at least two items from other categories. The items were segmented in blocks of six, and the blocks moved one position forward with each new subject. Each presentation sequence was used equally often in each condition.

Procedure. The subjects were tested individually. They were instructed to learn a list of action phrases for a later memory test. The items were presented visually. Half of the subjects were asked to learn the phrases by attentive reading (VT), and the other half were instructed to learn the items by performing the actions without using real objects - that is, by mimicking the actions (SPT). For half of the subjects in each condition, it was not mentioned that the phrases could be organized along categories. The other half of the subjects in each condition were informed in advance that the study list was categorically structured and the labels of the six categories were named. These subjects were also requested to use the categories during learning by internally assigning the items to the categories. The action phrases were presented one after the other on a computer screen. Each phrase was preceded by a warning tone and was displayed for $5 \mathrm{sec}$. After an interval of $500 \mathrm{msec}$, the next phrase appeared on the screen. After learning the phrases, the subjects were requested to write down all phrases they could remember in any order they liked. The free recall portion of the experiment lasted $4 \mathrm{~min}$.

\section{Results and Discussion}

The three different study lists had no differential effects on the results. Therefore, the data were collapsed over lists for further analyses. The data of the recall test are presented in Table 1. A phrase was scored as correct when the noun and the verb of the phrase were recalled correctly.

A $2 \times 2$ analysis of variance (ANOVA) with the variables encoding condition and preinformation yielded a main effect of encoding conditions $[F(1,104)=16.68$, $\left.M S_{\mathrm{e}}=0.0130, p<.001\right]$. The recall level after SPTs (.38) was higher than that after VTs (.29). Preinformation had no significant effect on free recall $(F<1)$. The interaction between the two factors was not significant $(F<1)$.

The ARC scores are summarized in Table 2. The ARC scores were analyzed in a $2 \times 2$ ANOVA with the variables encoding condition and preinformation. This analysis yielded a main effect neither for encoding condition $(F<1)$ nor for preinformation $(F<1.3, p>.25)$. How-

Table 1

Mean Proportion of Items Recalled (and Standard Deviations) as a Function of Encoding Condition and Preinformation

About the Categorical Structure of the List in Experiment 2

\begin{tabular}{llllll}
\hline \multirow{2}{*}{\multicolumn{1}{c}{ Preinformation }} & \multicolumn{2}{c}{ SPT } & & \multicolumn{2}{c}{ VT } \\
\cline { 2 - 3 } \cline { 5 - 6 } & $M$ & $S D$ & & $M$ & $S D$ \\
\hline Without preinformation & .38 & .12 & & .28 & .12 \\
With preinformation & .37 & .11 & & .29 & .11 \\
\hline
\end{tabular}

Note-SPT, subject-performed task; VT, verbal task.
Table 2

Mean Adjusted-Ratio-of-Clustering Scores (and Standard Deviations) as a Function of Encoding Condition and Preinformation About the Categorical Structure of the List in Experiment 2

\begin{tabular}{lllllll}
\hline \multirow{2}{*}{\multicolumn{1}{c}{ Preinformation }} & \multicolumn{2}{c}{ SPT } & & \multicolumn{2}{c}{ VT } \\
\cline { 2 - 3 } \cline { 6 - 7 } & $M$ & $S D$ & & $M$ & $S D$ \\
\hline Without preinformation & .43 & .33 & & .26 & .55 \\
With preinformation & .35 & .34 & & .52 & .38 \\
\hline
\end{tabular}

Note-SPT, subject-performed task; VT, verbal task.

ever, the two variables interacted $\left[F(1,104)=4.86, M S_{\mathrm{e}}=\right.$ $0.1670, p<.05]$. The interaction is due to the fact that the ARC scores of VTs increased from .26 without preinformation to .52 with preinformation $[F(1,104)=$ $5.66, p<.05]$. In SPTs, there was only a small and nonsignificant effect in the opposite direction, from .43 to $.35(F<1)$. ARC scores without preinformation were numerically higher in SPTs than in VTs, whereas with preinformation they were higher in VTs than in SPTs. When we tested these simple main effects in the ANOVA, the differences were not significant $(p<.12)$.

As was expected, the ARC scores for VTs and SPTsindicating the degree to which the categorical list structure influences the recall processes - interacted with preinformation about the categorical list structure.

The ARC scores for SPTs did not differ with and without preinformation. However, with preinformation about the categories, the ARC scores in VTs increased significantly. With preinformation, they were higher in VTs than in SPTs. The finding that explicitly communicating the category labels enhances the ARC scores in VTs but not in SPTs corresponds to our expectations. Enactment draws attention to the item to be performed and leaves little room for actively relating the presented items with each otherthat is, in SPTs the relational encoding processes are influenced very little by intentional processes. The VTs, in contrast, allow subjects to direct their attention to the categorical relations if the labels are explicitly given because there is no competing instruction, as there is in SPTs.

Moreover, although not significant, without preinformation about the categories the ARC scores were numerically even higher in SPTs than in VTs. That this effect was substantial but not significant (.17) was due to the fact that the variance in the VT condition without preinformation was high. We therefore took a closer look at the individual ARC scores in VTs. It turned out that 10 of the 27 subjects had zero or negative ARC scores - that is, less organization than would be expected by chancewhen no preinformation about categories was given. Thus, it might be that if a list structure is not salient, some subjects in VTs tend to organize the material along alternative structures to those intended by the experimenter. In this way, the mean ARC score of the VT group can be reduced.

The free recall level was not affected by preinformation about the categorical list structure. Such a null effect is plausible for SPTs if one assumes that SPT recall depends 
mainly on item-specific information and on distinctivenessbased retrieval, which makes it fairly independent of relational information (Seiler \& Engelkamp, 2003; Zimmer et al., 2000). In VTs, preinformation obviously increased relational information, and this may have increased searchbased retrieval processes. However, distinctiveness-based retrieval processes may have suffered (Seiler \& Engelkamp, 2003). Therefore, free recall did not change in VTs either.

The fact that free recall was better after SPTs than after VTs with and without preinformation may indicate that, as predicted, the large amount of item-specific information of SPTs, which makes free recall of SPTs largely independent of relational information, cannot be compensated in a VT even if relational information is better for VTs than for SPTs (Seiler \& Engelkamp, 2003).

\section{GENERAL DISCUSSION}

The main finding of the present study is that subjects in VTs benefit more from knowing the category labels in advance than do subjects in SPTs if list structures are not obvious, as is the case with a random presentation of list items. With such preinformation, ARC scores for VTs are higher than those for SPTs. This finding confirms the proposal that the corresponding numerical VT advantage in the study of Zimmer and Engelkamp (1989, Experiment 2) is not a chance result but reflects a reliable effect that can be observed with object-based taxonomic lists (present study, Experiment 2), with actionbased taxonomic lists (Zimmer \& Engelkamp, 1989, Experiment 2; present study, Experiment 1), and with episode-based lists (Zimmer \& Engelkamp, 1989, Experiment 2). It is in line with the assumptions that VTs allow better intentional relational encoding than do SPTs. As a consequence, the clustering effect is higher in VTs than in SPTs if subjects are given the category labels in advance and are requested to use them.

Evidence of the assumption that VTs allow for better intentional relational encoding than do SPTs has been presented before for unrelated items (see, e.g., Engelkamp, Mohr, \& Zimmer, 1991; Engelkamp \& Seiler, 2003; Olofsson, 1997). In these studies, it is assumed that enactment hinders the intentional building of interitem subjective associations between unrelated actions. Due to the performing instructions in SPTs, attention is focused on the individual items and thereby distracted from subjective relational encoding. In contrast, it is assumed that VTs allow for a more flexible allocation of attention to item-specific and relational encoding processes. Subjects in VTs get a less clear objective than do subjects in SPTs. The broad objective of retaining items allows active and flexible processing. Subjects in this task can search intentionally for subjective interitem associations. The possibility of intentional encoding of subjective associations of unrelated items has also been claimed by others (e.g., Craik, Govoni, Naveh-Benjamin, \& Anderson, 1996; Naveh-Benjamin, Hussain, Guez, \& Bar-On, 2003).
The main evidence for this view in research on action memory was seen in studies of paired-associate learning of unrelated actions. In free recall of single items, the usual SPT effect was observed. In contrast, in cued recall for the second action of a pair given the first as cue, the effect was reversed. Cued recall was poorer for SPTs than for VTs. This impairment was explained by the difficulty of associating unrelated actions under enactment (Engelkamp, 1986; Engelkamp et al., 1991).

The finding that VTs allow better intentional relational encoding of structured lists than do SPTs is also generally important beyond the field of SPTs because it shows that identical relational orienting tasks with identical study lists can be differentially effective under different task conditions - for example, if one task is verbal and one task is motor. In other studies, the orienting tasks were applied only in the context of verbal tasks (see, e.g., Hunt \& Einstein, 1981; McDaniel et al., 1988). In these studies, it was shown that the structure of word lists suggested a specific kind of encoding. A good structure induced relational encoding, whereas a poor structure induced item-specific encoding. These spontaneous kinds of encoding were shown to be modified by explicit orienting tasks for item-specific and relational encoding. We have been able to show that, beyond these studies, the modulating influence of a relational orienting task with a given list structure also depends on task modality. A task that requires subjects to organize the items along the categories affects relational encoding in VTs and SPTs differently.

The finding that preinforming subjects about the categories leads to higher ARC scores in VTs than in SPTs, whereas the SPT effect in free recall is unchanged, suggests, moreover, that categorical relational information does not contribute to the SPT effect. In Experiment 2 of the present study, we also replicated the repeatedly observed effect of no clustering differences (as measured by ARC scores) between VTs and SPTs (see, e.g., Engelkamp \& Zimmer, 1996; Norris \& West, 1993) if categorical lists are presented in a random order. However, numerically, clustering was even found to be higher in SPTs than in VTs, a result that has also sometimes been reported (e.g., Bäckman et al., 1986; Engelkamp et al., in press). The observation that the variance under this condition is larger in VTs than in SPTs can explain why substantial SPT advantages are sometimes significant (Bäckman et al., 1986; Engelkamp et al., in press, Experiment 2) and sometimes not (Engelkamp et al., in press, Experiment 1; present study, Experiment 2). The result likely depends on how strongly intentional processes in VTs deviate from list structure. In any case, the SPT advantage is considered to be a consequence of the intentional encoding processes in VTs. Due to accidental aspects of the list structure, intentional relational encoding processes in VTs may work against the use of category relational information, resulting in a systematic deviation of organizational processes from the categorical structure of the list. In contrast, in SPTs, such intentional relational encoding processes, which are inade- 
quate according to the intended relational encoding, do not emerge because subjects are forced to focus on single items. Hence, the particular results observed for ARCs with randomly arranged categorical relational lists depend on the balance between factors that work in favor and those that work against intentional category encoding.

In summary, altogether, our theoretical framework was not only confirmed by the present experiments, in which some central predictions were tested, but this framework is moreover able to explain and reconcile the inconsistent clustering findings from the literature and is consistent with similar considerations in related fields. Our framework makes assumptions similar to those made by Naveh-Benjamin (2000; Naveh-Benjamin et al., 2003) in his associative deficit hypothesis. Like us and others, he distinguished between item-specific and relational information. More important, however, is the fact that he assumed, as we do, that relational encoding can occur without intention as well as intentionally. Unintentional relational encoding is particularly important with unrelated items.

With regard to free recall, two findings of the present study are prominent. First, independent of all list and instructional variations, an SPT effect was consistently observed. Second, increasing the clustering by advance information about the categorical list structure did not increase the recall level.

The first finding is generally attributed to very efficient item-specific encoding of SPTs, which cannot be compensated by a better relational encoding of VTs. This finding is directly related to the second finding: that enhanced clustering in VTs did not enhance free recall. This finding is only partly in line with the literature. For instance, Gollin and Sharps (1988) observed that a blocked presentation of words of a categorically related list increased both clustering and free recall in comparison with a random presentation. The null effect of the present study can be explained differently for VTs and for SPTs. In SPTs, this null effect is plausible because the preinformation did not increase the ARC scores of SPTs (Zimmer \& Engelkamp, 1989, Experiments 1, 2, and 3; present study, Experiment 1). The situation is different for VTs. In the present study (Experiment 2), ARC increased in VTs with preinformation (from .26 to .52), but free recall did not (from .28 to .29). Corresponding results were observed in the study of Zimmer and Engelkamp (1989) if taxonomic lists were used (free recall, . 36 vs. .31; ARC, . 31 vs. .41), but recall increased in the case of episodic lists (free recall, .48 vs. .70; ARC, .52 vs. $.70)$. It seems as though clustering influences the recall level only if it is sufficiently high under random presentation. We speculate that in VTs the actual degree of clustering under random presentation determines the dominant retrieval strategy. That is, the actual retrieval strategies are determined by a subtle tuning, which is dependent on the degree with which item-specific and relational information is available.
The findings of the free recall performances are in line with the assumption that the relationship between free recall and clustering is complex and flexible. This fact makes it difficult to make predictions about the recall level. The complexity arises from the fact that itemspecific and relational information are not only variably encoded but that, in addition, the actual retrieval processes in free recall depend on the relative availability of both types of information. Strong item-specific information may lead to a dominance of distinctiveness-based retrieval processes, and strong relational information may favor search-based retrieval processes. It seems as though both kinds of retrieval processes are flexibly combined to yield an optimal recall level given the specific amounts of item-specific and relational information in each actual situation (see Seiler \& Engelkamp, 2003).

\section{REFERENCES}

Bäckman, L., Nilsson, L.-G., \& Chalom, D. (1986). New evidence on the nature of the encoding of action events. Memory \& Cognition, 14, 339-346.

BiEgElmanN, U. E. (1994). Relationale und itemspezifische Information: Untersuchungen zum Trade-off und zur Redundanz [Relational and item-specific information: Investigations into tradeoff and redundancy]. Frankfurt am Main: Haag \& Herchen.

BousfiELD, W. A. (1953). The occurrence of clustering in the recall of randomly arranged associates. Journal of General Psychology, 49, 229-240.

BURNS, D. J. (1993). Item gains and item losses during hypermnesic recall: Implications for the item-specific-relational information distinction. Journal of Experimental Psychology: Learning, Memory, \& Cognition, 19, 163-173.

Chalfonte, B. L., \& Johnson, M. K. (1996). Feature memory and binding in young and older adults. Memory \& Cognition, 24, 403416.

CoHen, R. L. (1981). On the generality of some memory laws. Scandinavian Journal of Psychology, 22, 267-281.

CoHEN, R. L. (1983). The effect of encoding variables on the free recall of words and action events. Memory \& Cognition, 11, 575-582.

Cohen, R. L. (1985). On the generality of the laws of memory. In L.-G. Nilsson \& T. Archer (Eds.), Animal learning and human memory (pp. 247-277). Hillsdale, NJ: Erlbaum.

Craik, F. I. M., Govoni, R., NAveh-Benjamin, M., \& Anderson, N. D. (1996). The effect of divided attention on encoding and retrieval processes in human memory. Journal of Experimental Psychology: General, 125, 159-180.

Einstein, G. O., \& Hunt, R. R. (1980). Levels of processing and organization: Additive effects of individual item and relational processing. Journal of Experimental Psychology: Human Learning \& Memory, 6, 588-598.

ENGELKAMP, J. (1986). Nouns and verbs in paired-associate learning: Instructional effects. Psychological Research, 48, 153-159.

ENGELKAMP, J. (1988). Modality-specific encoding and word class in verbal learning. In M. M. Gruneberg, P. E. Morris, \& R. N. Sykes (Eds.), Practical aspects of memory: Current research and issues (Vol. 1, pp. 415-420). Chichester, U.K.: Wiley.

ENGELKAMP, J. (1995). Visual imagery and enactment in memory of actions. British Journal of Psychology, 86, 227-240.

EngelKamp, J. (1998). Memory for actions. Hove, U.K.: Psychology Press.

Engelkamp, J., Biegelmann, U., \& McDaniel, M. A. (1998). Relational and item-specific information: Trade-off and redundancy. Memory, 6, 307-333.

ENGELKAMP, J., \& DEHN, D. (2000). Item and order information in subject-performed tasks and in experimenter-performed tasks. Jour- 
nal of Experimental Psychology: Learning, Memory, \& Cognition, 26, 671-682.

ENGelKamp, J., \& KRUMNACKeR, H. (1980). Imaginale und motorische Prozesse beim Behalten verbalen Materials [Imaginary and motor processes in memory for verbal material]. Zeitschrift für Experimentelle \& Angewandte Psychologie, 27, 511-533.

EngelKamp, J., Mohr, G., \& Zimmer, H. D. (1991). Pair-relational encoding of performed nouns and verbs. Psychological Research, 53, 232-239.

EngelKamp, J., \& Seiler, K. H. (2003). Gains and losses in action memory. Quarterly Journal of Experimental Psychology, 56A, 829848.

EngelKamp, J., Seiler, K. H., \& Zimmer, H. D. (in press). Memory for actions: Item and relational information in categorized lists. Psychological Research.

ENGELKAMP, J., \& Zimmer, H. D. (1983). Zum Einfluß von Wahrnehmen und Tun auf das Behalten von Verb-Objekt-Phrasen [The influence of perception and enactment on the retention of verb-object phrases]. Sprache \& Kognition, 2, 117-129.

ENGELKAMP, J., \& Zimmer, H. D. (1994). The human memory: A multimodal approach. Seattle: Hogrefe \& Huber.

EngelKamp, J., \& Zimmer, H. D. (1996). Organization and recall in verbal tasks and in subject-performed tasks. European Journal of Cognitive Psychology, 8, 257-273.

ENGELKAMP, J., \& ZimMER, H. D. (1997). Sensory factors in memory for subject-performed tasks. Acta Psychologica, 96, 43-60.

EngelKamp, J., \& Zimmer, H. D. (2002). Free recall and organization as a function of varying relational encoding in action memory. Psychological Research, 66, 91-98.

Gillund, G., \& Shifrrin, R. M. (1984). A retrieval model for both recognition and recall. Psychological Review, 91, 1-67.

Gollin, E. S., \& Sharps, M. J. (1988). Facilitation of free recall by categorical blocking depends on stimulus type. Memory \& Cognition, 16, 539-544.

Greene, R. L. (1992). Human memory: Paradigms and paradoxes. Hillsdale, NJ: Erlbaum.

Helstrup, T. (1987). One, two or three memories? A problem-solving approach to memory for performed acts. Acta Psychologica, 66, 3768.

HockLey, W. E., \& CRISTI, C. (1996). Tests of encoding tradeoffs between item and associative information. Memory \& Cognition, 24, 202-216.

HunT, R. R., \& EInSTEIN, G. O. (1981). Relational and item-specific information in memory. Journal of Verbal Learning \& Verbal Behavior, 20, 497-514.

Hunt, R. R., \& SETA, C. E. (1984). Category size effects in recall: The roles of relational and individual item information. Journal of Experimental Psychology: Learning, Memory, \& Cognition, 10, 454464.

KNOPF, M. (1991). Having shaved a kiwi fruit: Memory of unfamiliar subject-performed actions. Psychological Research, 53, 203-211.

KoRMI-NouRI, R. (1995). The nature of memory for action events: An episodic integration view. European Journal of Cognitive Psychology, 7, 337-363.

Kormi-Nouri, R., \& NiLsson, L.-G. (1999). Negative cueing effects with weak and strong intralist cues. European Journal of Cognitive Psychology, 11, 199-218.

McDaniel, M. A., DeLosh, E. L., \& Merritt, P. S. (2000). Order information and retrieval distinctiveness: Recall of common versus bizarre material. Journal of Experimental Psychology: Learning, Memory, \& Cognition, 26, 1045-1056.

McDaniel, M. A., Einstein, G. O., Dunay, P. K., \& Cobb, R. E. (1986). Encoding difficulty and memory: Toward a unifying theory. Journal of Memory \& Language, 25, 645-656.

McDaniel, M. A., Einstein, G. O., \& Lollis, T. (1988). Qualitative and quantitative considerations in encoding difficulty effects. Memory \& Cognition, 16, 8-14.

Mulligan, N. (1999). The effects of perceptual interference at encoding on organization and order: Investigating the roles of item-specific and relational information. Journal of Experimental Psychology: Learning, Memory, \& Cognition, 23, 54-69.
Mulligan, N. (2001). Generation and hypermnesia. Journal of Experimental Psychology: Learning, Memory, \& Cognition, 27, 436-450.

Naveh-Benjamin, M. (2000). Adult age differences in memory performance: Tests of an associative deficit hypothesis. Journal of Experimental Psychology: Learning, Memory, \& Cognition, 26, 11701187.

Naveh-Benjamin, M., Hussain, Z., Guez, J., \& Bar-On, M. (2003). Adult age differences in episodic memory: Further support for an associative-deficit hypothesis. Journal of Experimental Psychology: Learning, Memory, \& Cognition, 29, 826-837.

NiLsson, L.-G. (2000). Remembering actions and words. In F. I. M. Craik \& E. Tulving (Eds.), Oxford handbook of memory (pp. 137148). Oxford: Oxford University Press.

NoRris, M., \& West, R. (1993). Activity memory and aging: The role of motor retrieval and strategic processing. Psychology \& Aging, 8, 81-86.

Olofsson, U. (1997). Win some, lose some: Hypermnesia for actions reflects increased item-specific processing. Memory \& Cognition, 25, 797-800.

Ritchey, G. H. (1980). Picture superiority in free recall: The effects of organization and elaboration. Journal of Experimental Child Psychology, 29, 460-474.

Roediger, H. L., III, Balota, D. A., \& Watson, J. M. (2001). Spreading activation and arousal of false memories. In H. L. Roediger III, J. S. Nairne, I. Neath, \& A. M. Surprenant (Eds.), The nature of remembering: Essays in honor of Robert G. Crowder (pp. 95-115). Washington, DC: American Psychological Association.

Roenker, D. L., Thompson, C. P., \& Brown, S. C. (1971). Comparison of measures for the estimation of clustering in free recall. Psychological Bulletin, 76, 45-48.

Saltz, E., \& Donnenwerth-Nolan, S. (1981). Does motoric imagery facilitate memory for sentences? A selective interference test. Journal of Verbal Learning \& Verbal Behavior, 20, 322-332.

Seiler, K. H., \& ENGELKAMP, J. (2003). The role of item-specific information for the serial position curve in free recall. Journal of Experimental Psychology: Learning, Memory, \& Cognition, 29, 954964.

Zimmer, H. D. (1989). Visuelle und semantische Merkmale im Recall [Visual and semantic features in recall]. Sprache \& Kognition, 8, 115125.

ZIMMER, H. D. (1991). Memory after motoric encoding in a generationrecognition model. Psychological Research, 53, 226-231.

Zimmer, H. D. (2001). Why do actions speak louder than words? Action memory as a variant of encoding manipulations or the result of a specific memory system? In H. D. Zimmer, R. Cohen, M. J. Guynn, J. Engelkamp, R. Kormi-Nouri, \& M. A. Foley (Eds.), Memory for action: A distinct form of episodic memory? (pp. 151-198). New York: Oxford University Press.

ZimMER, H. D., \& EnGELKAMP, J. (1989). Does motor encoding enhance relational information? Psychological Research, 51, 158-167.

ZimMER, H. D., \& ENGELKAMP, J. (1999). Levels-of-processing effects in subject-performed tasks. Memory \& Cognition, 27, 907-914.

Zimmer, H. D., Helstrup, T., \& Engelkamp, J. (2000). Pop-out into memory: A retrieval mechanism that is enhanced with the recall of subject-performed tasks. Journal of Experimental Psychology: Learning, Memory, \& Cognition, 26, 658-670.

\section{NOTES}

1. The ARC score is defined as

$$
\mathrm{ARC}=\frac{[R-E(R)]}{[\max R-E(R)]}
$$

and is calculated on the basis of the recall protocol. It is the number of observed intracategory repetitions $(R)$ minus the expected number of intracategory repetitions $[E(R)]$ given these recalled items divided by the maximally possible number of intracategory repetitions $[\max (R)-$ $E(R)]$. Hence, it measures the extent to which subjects have arranged the items into their categories relative to the optimal reconstruction of categories that would have been possible given the items that were recalled. The possible maximum, $\max (R)$, is $N-k$-that is, the total number of 
items recalled minus the number of categories $(k)$ represented in the recall protocol. $E(R)$ is calculated as

$$
\left(\sum_{i}^{k} \frac{n_{i}^{2}}{N}\right)-1
$$

where $n_{i}$ is the number of items recalled from Category $i$ and $N$ is the total number of items recalled. The maximally possible ARC score is 1 , organization by chance should lead to an ARC score of zero, and negative values should occur if subjects consistently use relational structures other than the categories represented in the lists. The maximum nega- tive ARC score is not necessarily - 1. Depending on the opportunity for number of repetitions below chance, $\min (\mathrm{ARC})$ varies between zero and -1 (see Roenker et al., 1971, p. 47).

2. The typical serial position curve is masked by categorical relational information. Categorical encoding and retrieval conflict with the typical influence of serial positions. Therefore, we did not study the serial position curve here.

(Manuscript received March 27, 2002; revision accepted for publication June 21, 2004.) 